Revue européenne des sciences sociales

European Journal of Social Sciences

XXXIX-121 | 2001

L'acteur. Un concept sur la scène des sciences sociales

\title{
Acteur, agent, actant: personnages en quête d'un scénario introuvable
}

Jean-Claude Passeron

\section{CpenEdition}

\section{Journals}

Édition électronique

URL : http://journals.openedition.org/ress/643

DOI : $10.4000 /$ ress. 643

ISSN : 1663-4446

Éditeur

Librairie Droz

\section{Édition imprimée}

Date de publication : 1 novembre 2001

Pagination : 15-30

ISBN : 2-600-00663-X

ISSN : 0048-8046

\section{Référence électronique}

Jean-Claude Passeron, « Acteur, agent, actant: personnages en quête d'un scénario introuvable », Revue européenne des sciences sociales [En ligne], XXXIX-121 | 2001, mis en ligne le 11 décembre 2009, consulté le 19 avril 2019. URL : http://journals.openedition.org/ress/643 ; DOI : 10.4000/ress.643 


\section{ACTEUR, AGENT, ACTANT: Personnages en quête d'un scénario introuvable}

Existe-t-il, dans les sciences sociales, un langage théorique qui permette de parler plus scientifiquement que d'autres des actions qui s'enchaînent et se répondent dans une société?

Quand il s'agit de préciser le rôle que peut jouer Homo sociologicus sur la scène des théories scientifiques, les scénarios qu'en ont proposés les chercheurs ne manquent certes pas; ni les costumes variés des protagonistes possibles, ni les métaphores filées qui ont fait le succès des tirades classiques, ni les décors ou les accessoires méthodologiques qui désignent immédiatement à un public d'amateurs les auteurs bien catalogués de cette Comedia dell'arte en quête de ses personnages: le calculateur, le conteur, le détective, l'explorateur, l'improvisateur, l'abstracteur de quintessence, l'imprécateur, l'érudit, le prophète, etc... Mais aucun des scénarios sociologiques qu'ils proposent pour narrer le monde social ne s'accorde avec les autres livrets; les rôles n'y sont pas stabilisés comme dans un répertoire théâtral, qui pourrait figurer pour les sciences sociales ce que sont une théorie, un «paradigme» ou un «programme» scientifiques dans les sciences exactes. L'intrigue qui noue les interactions sociales est écrite par chaque école sociologique dans un langage différent qui impose aux interprètes une ré-interprétation des répliques échangées par les acteurs; chaque dramaturge compose son texte pour imposer sa langue de description, bien souvent son dialecte et parfois son jargon, lesquels, bien évidemment, redéfinissent le sens des actions et des passions des personnages, redistribuent les premiers et les seconds rôles. On est fondé à parler d'imbroglio scientifique pour désigner le foisonnement des langages théoriques qui nourrit la discordance des écoles et des styles d'analyse toujours concurrents ou guerroyant dans l'histoire des sciences de l'homme.

Quelques uns de ces langages possèdent cependant une prégnance linguistique qui a marqué et marque encore l'histoire de nos disciplines, comme on le voit si on la mesure à la fréquence d'apparition des termes de son lexique et des schèmes caractéristiques de son raisonnement dans les textes des chercheurs en sciences sociales. Est-ce la robustesse théorique de certains langages conceptuellement mieux forgés que d'autres ou la facilité de les imiter, est-ce leur force théorique de preuve ou bien leur pouvoir métaphorique de suggestion qui explique l'ampleur de leur diffusion et leurs retours sur le devant de la scène scientifique, même après des périodes d'obsolescence ou de discrédit? On peut se poser la question pour les deux métaphores de l'action sociale qui me semblent avoir été les plus largement utilisées: celle de «l'acteur» jouant un rôle sur une «scène sociale» et celle de «l'agent» supposé être le porteur interchangeable des exigences d'un «système». J'y ajouterai volontiers un troisième langage de l'analyse des actions, celui de 
«l'actant», corrélatif d'une tout autre conception du «système». Bien que d'un emploi plus récent et plus spécialisé, ce concept éclaircit peut-être, sur un cas particulier, le point de discordance entre les deux autres, en utilisant pour décrire un système (le langage en l'occurrence) la description de l'activité symbolique de celui qui l'utilise (dans la «parole»), décrivant, du même coup, l'activité de cet actant linguistique comme une action qui fonde les catégories sémantiques et les opérations syntaxiques inscrites dans la structure du système.

\section{DISPOSITIF THÉORIQUE ET MÉTAPHORIQUE DES THÉORIES DE L'ACTION.}

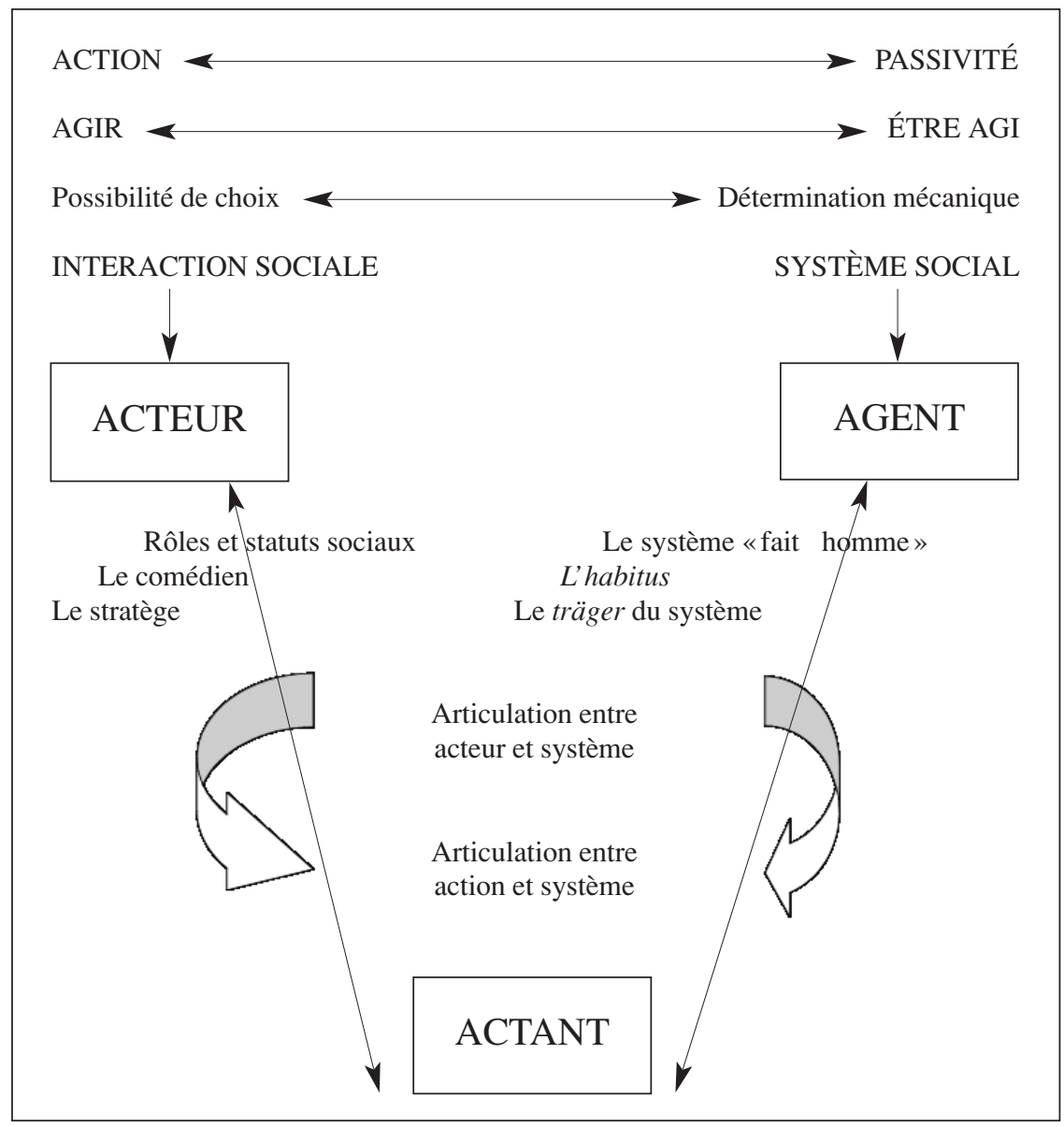

Les flèches pleines à une pointe soulignent un engendrement entre concepts ou représentations; à double pointe leur opposition. Les flèches fines à double pointe une articulation entre deux concepts qui oblige à les définir l'un par l'autre dans un programme de recherches. 


\section{LA MÉTAPHORE DE LA SOCIÉTÉ COMME THÉÂTRE}

\section{All the world's a stage and all the men merely players...}

C'est l'incipit d'une tirade shakespearienne qu'on voit souvent citer dans les introductions de manuels américains de sociologie pour présenter la théorie des «rôles» et «statuts » sociaux et en tirer une définition immédiatement compréhensible de l'objet propre à cette discipline. «Les âges de la vie» qu'énumérait Shakespeare parlant d'un «théâtre du monde» sur lequel tous les hommes viennent successivement jouer leurs rôles biographiques - depuis le rôle du nourrisson «bavant et rampant comme un escargot» jusqu'à celui du vieillard édenté et rabâcheur - proposent une métaphore suggestive de la contrainte qu'exerce un «rôle» sur celui qui l'interprète. Comme en toute régulation d'une interaction sociale, il s'agit d'une obligation interne que le «comédien» ressent comme une prise de décision inspirée par son désir personnel d'action, enracinée dans le sens vécu de ses représentations et croyances propres, mais qu'il subit aussi comme une contrainte externe, imposée par le texte de l'auteur et les attentes des spectateurs. La théorie des «rôles sociaux» propose une définition de la liberté de jeu de «l'acteur social», qui fournit en même temps au sociologue attaché à l'explication des comportements par des choix individuels le principe de toutes les régularités, conformités et continuités sociales.

\section{Statuts, rôles, attentes}

Dans un tel langage l'explication sociologique est à la fois générale et individualisée: c'est parce qu'un individu occupe un «statut social», assigné ou acquis» (ascribed ou achieved ), qu'il endosse un «rôle qui lui impose des comportements «modelés» par les «attentes» (expectations) des autres acteurs sociaux à l'égard de tout individu qui occuperait ce même statut. Schématiser ainsi ce qu'est la normativité inhérente à toute vie sociale, en se guidant sur le modèle des règles d'exécution d'un rôle dictées à un comédien par un texte figurant dans un répertoire, est beaucoup plus qu'user d'une simple métaphore. En se systématisant comme théorie, le langage analogique de l'acteur social a engendré une grille de description capable de conceptualiser, dans les domaines les plus divers de l'action sociale, l'ensemble articulée des «modèles de comportement» qui font la cohésion d'une société, la cohérence de sa culture, la compatibilité des attentes réciproques entre les acteurs qui y interviennent, assurant ainsi la régulation et la régularité de toutes les «interactions» qui trament la vie d'une société. Depuis G. H. Mead qui en avait formulé la psychologie sociale, cette théorie de la communication et de l'intégration sociales s'est étendue et développée dans la sociologie américaine et anglo-saxonne, puis au-delà, en transférant son intelligibilité descriptive et explicative globale à toute une gamme de «théories de la société » ${ }^{1}$. On peut même dire qu'à partir des années ' 50 ' sa généralité a tendu à

\footnotetext{
Nous voulons souligner par cette distinction entre «théorie de la société » et « théorie de la connaissance sociologique », la portée du langage de « l'acteur » et de la « scène », qui est davantage qu'une théorie particulière de la société : c'est un langage général de la formulation des théories particulières (cf. la distinction entre les $\mathbf{T} \mathbf{1}$ et les $\mathbf{T} \mathbf{2}$ in J.-C. Passeron, « De la pluralité théorique en sociologie », Revue européenne des sciences sociales, Genève, Droz, n99, 1994.
} 
en faire un espéranto de la sociologie mondiale - en tout cas de celle des Congrès mondiaux de sociologie. R. Dahrendorf pouvait, dans les années ' 60 ' résumer en quelques mots la prétention à l'universalité de ce langage sociologique: «L'homo sociologicus c'est l'homme qui occupe un statut et joue un rôle conformément aux attentes des partenaires qui entrent en interaction avec lui dans un même système de rôles ». Cette théorie, au sens T 1, s'est prolongée, en particulier en économie, en sociologie et en ethnologie, en fournissant un cadre conceptuel à de nombreux «programmes» de recherche tels que ceux de «l'individualisme méthodologique», de «l'interactionnisme symbolique», du «culturalisme», de la «construction du social» ou même de «l'ethnométhodologie», s'associant parfois à d'autres langages théoriques plus éloignés qui la mettaient en relations avec les descriptions macro-sociologiques du système ou de la fonction, par exemple dans le structuro-fonctionnalisme de Parsons,.

Si l'on se reporte au schéma ci-dessus - qui situe les choix explicatifs des sciences sociales entre le pôle d'une explication des régularités sociales par la libre activité des acteurs individuels et le pôle de leur explication par les mécanismes d'un système global dont la structure détermine le comportement anonyme d'agents interchangeables sans qu'ils aient à en décider - on voit que le langage de l'acteur social a rencontré, jusqu'à la seconde guerre mondiale, son principal adversaire intellectuel dans le langage alternatif d'explication des phénomènes sociaux qu'utilisaient la plupart des traditions scientifiques européennes. En France et en Allemagne en particulier, les sciences de l'homme s'étaient fondées dans le prolongement de sciences historiques, dotées par leur passé philosophique et scientifique de la certitude de détenir une forme supérieure d'intelligibilité, qui n'appartient qu'aux systèmes universels d'explication. Le langage psychologique du «jeu social», du faire-semblant, a, bien sûr, des antécédents et des représentants glorieux dans l'histoire des idées européennes. Mais du XVI ${ }^{\mathrm{e}}$ au XVIII ${ }^{\mathrm{e}}$ siècle, c'est la tradition des «moralistes» qui l'a cultivée, à des fins polémiques ou apologétiques, pour dénoncer les effets imaginaires de la «grimace sociale » dans les cérémonies juridiques ou les mises en scène de l'apparat politique, démystifier le respect des «grandeurs d'établissement» au détriment des «grandeurs naturelles» (Pascal), ou pour stigmatiser, au XVIII ${ }^{\mathrm{e}}$ siècle, l'hypocrisie sociale qui dissimule «l'amour propre» ou l'intérêt sous le masque de l'altruisme ou derrière les codes de civilité. Proche d'un scepticisme radical ou, au moins, d'un «soupçon» porté sur le sérieux ou la moralité des actions humaines dans l'histoire et la société, cette anthropologie pessimiste de la «connaissance du cœur humain » n'a favorisé directement ni la naissance ni le développement des sciences sociales proprement dites. Sur le continent, les fondateurs des grands systèmes sociologiques, économiques ou politiques ont plutôt puisé leurs concepts et leurs schèmes de pensée dans les textes de précurseurs de l'explication systémique tels que les physiocrates pour l'économie ou Montesquieu pour la sociologie, plus généralement dans les valeurs rationalistes d'une philosophie du

pp.78-90). La théorie des rôles est une «théorie de la connaissance sociologique » comme l'est aussi la théorie durkheimienne du « fait social » défini comme « institution » qui s’y oppose point par point : marxisme, durkheimisme, wébérianisme, théorie des « rôles » ou « individualisme méthodologique », « constructivisme » sont toutes des $\mathbf{T} \mathbf{1}$ qui formulent un ou plusieurs principes d'intelligibilité communs à de nombreuses T 2. 
progrès, dans les valeurs d'une morale philanthropique, dans une science économique des bilans et des calculs statistiques, dans une sociologie des crises et des équilibres collectifs. Les fondations théoriques des nouvelles sciences sociales, comme leurs choix de méthodes, se sont opérés, au XIX ${ }^{\mathrm{e}}$ siècle dans toute l'Europe continentale dont la pensée historique se sépare alors de l'utilitarisme anglais - chez Comte ou Marx, Durkheim, Pareto ou Weber, Nietzsche ou Croce - à partir de l'histoire globale et de ses civilisations, de ses typologies et de ses conflits, et presque toujours à partir de catégories de description et d'analyse faites pour appréhender les phénomènes collectifs.

\section{L'acteur ou le système?}

Y aurait-il aujourd'hui et partout dans le monde un «retour de l'acteur»comme on s'en félicite chez les sociologues l'action, Touraine par exemple, depuis les années ' 80 '» - qui se serait déclenché à la faveur de l'effondrement ou de l'usure des grands paradigmes » marxistes, structuralistes, fonctionnalistes et évolutionnistes dans les sciences sociales à partir des années'70'? Ce constat d'un Grand Retour des théories sociologiques du choix individuel, proscrites par l'intolérance des doctrinaires d'un déterminisme macro-sociologique, supposerait que les théories qui mettent l'acteur au principe de l'action sociale aient été durablement éclipsées, depuis la seconde moitié du XIX $\mathrm{X}^{\mathrm{e}}$ jusqu'au tournant du XX $\mathrm{XX}^{\mathrm{e}}$ siècle, par la diffusion des théories fonctionnalistes ou structuralistes liées à la plupart des grands systèmes sociologiques, y compris aux Etats-Unis dans la génération de Parsons et Merton. Ce n'est peut-être chez moi qu'une simple impression rétrospective mais je dirais plutôt que, si l'on s'en tient au fond et non aux mots, «l'acteur» n'a jamais vraiment quitté la scène sociologique.

Dans toutes les sciences sociales, le flux et le reflux des langages scientifiques m'ont toujours semblé aussi rapides que déconcertants - preuve sans doute qu'ils relèvent bien de l'ordre de l'invention scientifique: c'est dans les débats menés dans l'espace public ou sur la scène des médias, et non dans les pratiques et travaux du terrain, de l'archive ou de l'enquête, que le sociologue pourrait déceler, et presque prévoir, les changements d'humeur du grand public intellectuel en se guidant sur le modèle mécanique des retours périodiques du balancier. En matière d'histoire des idées scientifiques l'évaluation des mouvements à court terme suscite facilement, chez les contemporains, illusions d'optique ou lacunes d'inventaire: en qualifiant de «mode» ou d' «idéologie» la domination d'une théorie, on se donne un moyen commode de saluer le «revival» d'une autre dès que la première reflue, sans qu'on sache si c'est pour longtemps. La prévision de l'avenir scientifique d'une théorie ne peut guère se justifier par l'examen sociologique de ses premiers résultats, pas plus qu'une croissance ou une crise ne se prévoient vraiment à partir des calculs d'un modèle économique, ou un succès géopolitique à partir de l'analyse sociologique d'un processus en cours. Bien que largement répandue, souvent par les chercheurs déçus d'une doctrine dont ils avaient trop attendu, la thèse de l'obsolescence des théories fondées sur l'efficacité impersonnelle des structures ne me paraît pas mieux établie que la thèse d'une victoire définitive des théories qui prennent pour principe d'explication historique la liberté de choix d'un sujet individuel, psychologique ou rationnel: les deux langages d'interprétation et d'explication n'ont jamais cessé d'affronter leurs méthodes et leurs 
résultats, leurs intelligibilités et leurs programmes. Passons donc, sans plus épiloguer sur l'avenir scientifique de l'une ou de l'autre, à la deuxième métaphore de la causalité sociologique.

\section{LA MÉTAPHORE DE LA CONTRAINTE SOCIALE COMME MACHINERIE DU SYSTÈME}

Peut-être plus ancienne, si l'on remontait jusqu'à l'Antiquité le cours de son passé philosophique, la représentation selon laquelle le cours du monde historique est déterminé invisiblement par une ou des lois de nécessité ou de fatalité s'est trouvée amplifiée à l'époque moderne par les révolutions scientifiques en chaîne qui, dans toutes les sciences de la matière, ont dépossédé dès le XVI ${ }^{e}$ siècle la théologie de son pouvoir de définir le statut ontologique des explications et des causalités.

\section{Le système social et l'organisme}

Dans toutes les sciences de l'homme, la métaphore du mouvement des rouages dans un système mécanique a donné, dans le sillage de la nouvelle physique mathématique, sa première forme conceptuelle (au XVII siècle dans le cartésianisme ou chez Hobbes, puis Spinoza) à l'explication des mouvements de l'âme et à la recherche des fondements naturels du contrat social et politique. La métaphore du système comme machinerie - dont l'efficacité oblige le descripteur à restituer les mécanismes d'un dispositif d'interdépendances au fonctionnement d'ensemble du système pour y trouver une raison intelligible de ses effets - a encore renforcé, dans les sciences sociales, le schéma d'une stricte dépendance des «parties» par rapport à un «tout» en y agrégeant les pouvoirs d'une autre métaphore, venue des sciences de la vie, celle de la représentation de la société comme organisme.

En empruntant à la biologie les concepts fondamentaux de ses descriptions de fonctions et de structures sociales pour décrire «l'intégration», la «coopération» ou «l'adaptation» d'un groupe à son environnement externe ou celle des «parties» d'une société à un «tout» régulateur de son milieu interne et en les définissant par analogie avec la définition anatomique et physiologique de ces concepts dans un organisme, les sciences de la société ont renforçé, du XVIII ${ }^{e}$ au XIX ${ }^{e}$ siècle, pour le meilleur et pour le pire, l'emprise du modèle qui explique tout processus historique et toute interdépendance sociale par les lois d'un «système». Le système d'un organisme vivant détermine le sort de ses éléments plus implacablement encore qu'un système physique dépourvu de «finalité interne» et par là d'une stratégie auto-gouvernée susceptible de répondre aux dérèglements ou aux chocs. La métaphore du «système vivant» suggère une causalité sociologique plus systématique encore que celle d'un système mécanique puisque le fonctionnement biologique d'un organisme capable d'agir comme gestionnaire de sa propre totalité s'exprime essentiellement dans l'auto-régulation d'une totalité obéissant à une «finalité interne» qui subordonne les «parties» au maintien, ou au ré-équilibrage, d'un équilibre avec son milieu externe et externe. Tous les fonctionnalismes sont issus de cette analogie, ou plutôt de son application mécanique 
à la structure de tout système social; la force de l'analogie a longtemps dissuadé les sociologues d'observer les phénomènes qui auraient révélé son inadéquation descriptive. En transposant du vivant au social le «principe d' homeostatis» (Cannon), l'hypothèse d'une «unité fonctionnelle» inhérente à toute société efface presque toutes les propriétés spécifiques des sociétés et de leur histoire, et d'abord ses antagonismes internes.

Dans une deuxième vague métaphorique, la diffusion de la théorie darwinienne de l'évolution des espèces a redoublé, à la fin du XIX ${ }^{\mathrm{e}}$ siècle, l'influence de l'analogie biologique dans les sciences sociales en lui conférant une dimension historique. On en voit la portée dans le développement multiforme de l'«évolutionnisme social» qui, plus que «l'organicisme» ou les théories de l'hérédité et de la race, limités à quelques écoles, a marqué toutes les théories anthropologiques sociologiques, historiques ou même économiques, jusqu'à ce que, tard dans le $\mathrm{XX}^{\mathrm{e}}$ siècle, ses certitudes analogiques soient remises en question par la victoire du «relativisme culturel » en ethnologie, qui ne s'est étendu que progressivement aux autres sciences sociales. La composante biologique de l'évolutionnisme social a favorisé, y compris dans les recherches historiques fondées sur la méthode comparative, en tout cas dans leurs paraphrases philosophiques, une sociologie holiste et nomologique, où se sont longtemps confondues la recherche d'un «sens de l'histoire» et la recherche des «lois universelles» de l'évolution des sociétés. C'est l'application du schéma darwinien qui a imposé sa formulation nomologique à la plupart des programmes de recherche issus des sciences sociales au $\mathrm{XIX}^{\mathrm{e}}$ siècle: l'analogie biologique faisait coup double en suggérant la coexistence d'une loi de reproduction des formes sociales à l'identique (comme dans l'engendrement) et d'une direction de l'évolution historique qui ne pouvait être ordonnée (comme dans l'évolution des espèces) que selon une loi de progrès. L'évolutionnisme social a eu son Manifeste avec la «Loi des trois états » d'Auguste Comte, aujourd'hui surannée; mais la force persuasive de cet évolutionnisme se retrouvera encore, sous une forme positiviste à peine atténuée - tant elle est lié aux schèmes d'intelligibilité qui ont présidé à la naissance des sciences sociales - bien après Marx et sa théorie de «lois de l'histoire» qui, disait-il, se réalisent «aussi implacablement» que les lois darwiniennes de l'évolution, par exemple dans l'évolutionnisme durkheimien qui ordonne les sociétés et les formes de sociabilité sur un axe unique allant du «simple» au « complexe», ou dans le traitement wébérien de l'histoire des «rationalisations» et du «désenchantement du monde», et surtout pendant longtemps dans toutes les ethnologies du «primitif» ou de «l'archaïque », etc. Les schèmes de l'évolutionnisme social ont survécu à la révolution «relativiste» au moins par morceaux dans de nombreuses explications de science sociale, par exemple dans les théories classiques de la croissance économique ou de la rentabilité technique d'une innovation entendue comme raison suffisante de son adoption dans l'histoire économique.

\section{Degrés d'accentuation des métaphores; l'agent et l'acteur, le portefaix et le stratège}

Sous la forme développée d'une conception à la fois diachronique et synchronique du développement des sociétés et de la détermination mécanique du comportement de ses «agents », la métaphore du «système», conçu à la fois comme 
une machinerie imposant inexorablement ses contraintes aux rouages qui la composent et comme la loi d'un «organisme» gouvernant le fonctionnement de ses organes selon sa finalité interne, a irrigué et irrigue encore, souvent à leur corps défendant, l'intelligibilité de nombreuses théories sociologiques (ou parties de théories) contemporaines, y compris celles qui en récusent explicitement le passé scientiste. La robustesse et la longévité de cette $\mathbf{T} \mathbf{1}$ capable de susciter sans cesse des T $\mathbf{2}$ modernisées (après le positivisme comtien ou l'évolutionnisme spencérien, le marxisme, l'ethnologie de la «pensée primitive», puis le structuralisme, les rejetons ne manquent pas) qui ont permis des avancées empiriques et des percées théoriques dans toutes les sciences sociales, montrent - comme dans le cas de la T 1 qui fonde son intelligibilité sur celle des choix d'un «acteur» supposé auteur raisonnable et raisonneur de la rationalité de ses actes dans l'individualisme méthodologique - qu'aucun paradigme n'a jamais pu gouverner entièrement le champ de l'intelligibilité scientifique dans les sciences sociales. Plutôt que de détailler les «faits» que le langage de l'une laisse échapper alors que l'autre dispose du langage qui les lui fait voir, et vice versa, il est plus intéressant, pour juger de leur fécondité différentielle, de hiérarchiser, comme des degrés d'accentuation (ou d'exagération) de chacune des deux métaphores, les variantes du schème de la liberté de choix prêté à l'acteur et celles du schème de la toutepuissance des structures.

Comme l'indique le schéma qui présente deux gradations parallèles, on peut classer (1) à gauche, les langages dérivés de la théorie individualiste de l'action et de l'interaction, en allant du langage des rôles sociaux qui concède à «l'acteur» une liberté de jeu limitée par les attentes d'autrui, et que symbolise la figure du «comédien », en allant jusqu'à la liberté de supputer et calculer (sous contraintes d'information et de ressources) son utilité individuelle, maximale ou optimale, liberté qui définit l'espace de choix du «stratège» rationnel dans l'économie, la guerre, la concurrence sociale ou la théorie des jeux. On peut alors hiérarchiser à droite (2) les langages qui expliquent l'interdépendance ou la succession des faits sociaux en ne se donnant que des «agents » qui sont plus complètement agis par les lois d'un système à mesure que l'on va d'un simple encadrement des psychologies individuelles par les contraintes liées à la position des agents dans un système - c'est la définition par Marx du «capitaliste comme capital fait homme ${ }^{2}-$ vers des formulations plus dures de la possibilité de déduire la subjectivité psychologique de l'agent, sa personnalité individuelle, à partir des institutions, des structures ou des systèmes de normes qui lui préexistent - c'était la définition durkheimienne de la socialisation; c'est chez Bourdieu, la définition de l'habitus comme «intériorisation » des structures objectives de l'expérience sociale et principe de leur reproduction sociale par les pratiques qui «l'extériorisent» - en allant, par oubli croissant des individus, jusqu'aux formulations les plus «anti-

Dans ce passage du Capital, comme dans d'autres textes historiques, Marx emploie des formulations qui laissent une place à la description anthropologique. Le « capital fait homme » est encore un homme dont il faut expliquer les choix non pas seulement en fonction de son rôle d'agent du système, mais en fonction du contexte social qui fait par exemple (dans le passage cité sur l'héritier du capitaliste de première génération) que «deux hommes se disputent désormais son cœur » de bourgeois : le ré-investisseur ascétique du profit dans l'entreprise et le jouisseur dépensier, qui aspire à imiter le gaspillage ostentatoire de l'ancienne aristocratie pour affirmer son rang. 
humanistes» (comme disait Althusser) dans les structuralismes militants, qui, dans les années '60', frôlaient avec plus de plaisir philosophique que de conviction sociologique, l'idée d'une «mise à mort du sujet» par les méthodes expéditives du structuralisme dans les sciences de l'homme. Les analyses de la «fonction-auteur» par Foucault ou celles des ouvrages qu'il a consacrés aux «nappes discursives » rendues à leur signification historique par l'épouillement de leurs noms propres et de leurs anecdotes, retrouvaient paradoxalement en ce point extrême de la «signification faite structure» d'autres formulations de ce Marx qu'il n'aimait pas - et qui dans les moments les plus abstraits de son raisonnement causal pouvait même évacuer la métaphore de «l'agent» pour ne plus avoir à considérer qu'un «träger» de la structure, un simple «porteur» ou portefaix d'une efficacité qui ne lui doit rien: un x1 en somme, comme celui qui, en logique formelle, transforme une fonction propositionnelle $\mathbf{f}(\mathbf{x})$ (signifiant par exemple «marxiser») en une proposition vraie, s'il existe un x1 (l'individu Karl Marx par exemple) qui la rend susceptible d'être dite vraie ou fausse.

On aura remarqué que les deux gradations de programmes scientifiques de recherche aboutissent toutes les deux à des formalismes également éloignés des besoins des sciences sociales définies comme sciences empiriques et historiques: le formalisme des théories et des modèles mathématiques de l'économie pure d'un côté, le formalisme logico-mathématique des structuralismes les plus aventuriers de l'autre. On pourrait examiner - mais ce serait refaire toute l'histoire de nos disciples - les juxtapositions, les compromis, ou les articulations entre les langages de nos deux T1, sans parvenir à en extraire une vertu intrinsèque d'intelligibilité, qui autoriserait une préférence scientifique pour l'un ou l'autre. On ne peut même pas prétendre que les juxtapositions les plus naïves d'éléments empruntés à ces deux langages valent mieux, ou moins, que les compromis qui cachent des habiletés d'orateurs ou des éclectismes utiles à la carrière universitaire. Ni même que les articulations conceptuelles les mieux pensées entre «l'acteur et le système» fourniraient de meilleures armes théoriques au chercheur qu'un rattachement de commodité instrumentale à l'une ou l'autre, ou l'une et l'autre, selon les chantiers, les hypothèses ou les stades d'avancement d'une recherche. On découvre, en fait, que c'est l'adaptation des «programmes d'enquête» aux questions que l'on pose à un matériau historique qui décide de la valeur scientifique d'une recherche; et que la valeur heuristique de ces programmes tient à la cohérence sémantique des liens qu'ils instaurent entre un langage théorique et le langage de ses protocoles de mise à l'épreuve empirique, beaucoup plus qu'à la cohérence philosophique ou à la formalisation logique du langage théorique qu'ils utilisent.

\section{L'ACTANT COMME PRINCIPE DE DESCRIPTION D'UN SYSTÈME EN ACTE}

La dichotomie saussurienne entre «langue » et «parole » a fourni, avec la théorie du «signe», la base théorique de la linguistique et de la sémiologie modernes, autrement dit une définition opératoire de «l'objet» propre à une science générale des «systèmes de signes », dès lors qu'on pose avec Saussure, que «c'est le point 
de vue qui fait l'objet $»^{3}$. Mais chez Saussure comme chez ses successeurs on ne s'est guère prononcé pendant longtemps sur le sort scientifique qu'il convient de réserver à l'activité symbolique de l'acteur qui assure le passage de l'une à l'autre de ces deux formes d'existence de la signification, l'une virtuelle l'autre actuelle. L'instance de médiation entre les deux «états», l'un inerte, l'autre actif, de la signification, qui peut seule faire circuler du sens dans une communication entre des interlocuteurs, n'est complètement descriptible ni dans une linguistique de la langue, ni dans une linguistique de la parole. Tant qu'on les traite séparément et qu'on ne fait pas de l'acte d'énonciation lui-même l'acte fondamental de référence pour comprendre le sens de tous les énoncés possibles d'une langue, on ne tire pas de ce concept un programme de description linguistique, comme le fera Benvéniste. En posant que le discours «n'est que la langue en tant qu'elle est assumée par l'homme qui parle», Benvéniste n'a pas proposé d'introduire une médiation théorique de plus, qui opérerait seulement dans le discours méta-linguistique du commentateur ou du philosophe, mais il a construit sur cette base un programme empirique de description de l'actant symbolique: désormais toute analyse du fonctionnement des termes et des énoncés, dont le sens n'est mis en œuvre que dans le déroulement d'un discours, suppose sa théorie du rôle de l'énonciation entendue comme contexte référentiel du sens des énoncés ${ }^{4}$.

Comme le commentait Greimas ${ }^{5}$, qui a conçu sa sémiotique générale sur cette base, seul le programme d'Emile Benvéniste permet d'articuler une linguistique et une pragmatique des modes et registres discursifs en référant la détermination de leur sens à l'instance de leur énonciation. Par là, ce programme se distingue radicalement des philosophies ou des phénoménologies qui introduisent à cette place un «Je» transcendal ou un «moi » psychologique. «L'actant symbolique n'est pas un sujet ontologique, qui s'écrirait: l'homme qui parle; mais un concept grammatical: l' homme qui parle, traditionnellement connu en linguistique sous le nom de sujet parlant. (...) Le sujet du discours n'est, sémantiquement parlant, qu'une instance virtuelle, c'est-à-dire une instance construite dans le cadre de la théorie linguistique pour rendre compte de la transformation de la forme paradigmatique en une forme syntagmatique du langage $»^{6}$.

Autrement dit, dans le cas du langage, «l'actant» qui actualise le «système» est le principe même d'une action sans laquelle le système n'existerait pas, n'offrirait aucune prise à la description linguistique, et encore moins à une explication de l'interdépendance entre ses mécanismes de fonctionnement. «L'actant» n'est pas, face au système, un «acteur» libre de l'utiliser, de l'interpréter ou de le remanier comme un instrument, ni un simple «agent» d'actualisation qui pourrait être déduit des exigences du système, ni, encore moins, un usager extérieur déterminé par les exigences d'un autre système social et dont la rencontre avec le système linguistique pourrait être contingente: l'actant est inscrit dans la structure même du système à la fois comme condition et comme conséquence du pouvoir de signi-

F. de Saussure, Cours de linguistique générale (1915), Ed. T. de Mauro, Paris, Payot, 1974, p. 38.

Cf. E. Benvéniste, Problèmes de linguistique générale, Paris, Gallimard, 1966, en particulier la partie intitulée «L'homme dans la langue », pp. 225-286.

A. J. Greimas, Sémiotique et sciences sociales, Paris, Le Seuil,, 1976, pp. 9-42.

Ibidem 
fier propre à un système particulier de signes. Seul auteur possible de discours, il est l'opérateur de toutes les catégories sémantiques et de toutes les opérations syntaxiques qui sont possibles dans le système et dont la définition linguistique ne peut préexister à leur mise en œuvre dans un discours. «Le sujet du discours est donc cette instance qui ne se contente pas, selon la conception saussurienne, d'assurer le passage de l'état virtuel à l'état actuel du langage: il apparaît comme l'endroit où se trouve monté l'ensemble des mécanismes de la mise en discours de la langue. Situé en un lieu où l'être du langage se transforme en un faire linguistique en transmettant du sens, le sujet du discours peut être dit, sans que cela soit une mauvaise métaphore, producteur du langage $»$.

Une «action» ou une «interaction» fournissent un objet à une science de l'homme dans la seule mesure où leur «sens » peut faire l'objet d'une description empirique menée selon une méthode d'enquête. Ce n'est pas seulement, par exemple, pour comprendre le sens du système pronominal d'une langue que je dois ancrer «pragmatiquement» le lexique et la grammaire des pronoms dans le système de cette langue. Je ne puis comprendre le fonctionnement de toutes les catégories sémantiques et syntaxiques de cette langue comme système, son équilibre ou sa force de retour à l'équilibre, sans examiner les situations de parole et d'interlocution où son système pronominal est mis en œuvre, en même temps que son système de référentiels et de déictiques. Si je puis parler de choses et d'autres c'est que je sais ce que parler de quelqu'un à quelqu'un «veut dire». Si je puis parler et être entendu, c'est que je sais que je parle en tant qu'un «je» parlant à un «tu» pour lui parler d'un «il», qui ne sont pas à la même place pendant que je discours.

On a donc bien affaire avec le concept d' «actant» à un concept descriptif capable de fonder le programme empirique d'une description de processus singulier - ici le fil syntagmatique du discours dans le déroulement de la parole- programme qui articule alors étroitement les interactions sémantiques entre la structure d'un système et les choix de ses utilisateurs, comme entre toutes les opérations qui sont possibles dans la syntaxe du système. Si l'on s'en tient au cas assez particulier des sciences sémiotiques, on voit que ce langage savant de la description des phénomènes de signification et de communication - d'ailleurs peu suspect, en son aridité conceptuelle, de se prêter aux dérives suggestives de la métaphore - a ouvert de nouveaux horizons à toutes les disciplines linguistiques en aménageant un lien entre la «linguistique structurale» ou «générative» (sous ses diverses formes) et la«pragmatique» des situations de parole (sous ses diverses formes aussi). Il articule théoriquement les rapports intelligibles entre processus et système, dans une explication qui est à la fois historique et structurale, singulière et systématique. Il propose en tout cas une formule de «l'action sociale» moins limitative et mieux unifiée que la formule théorique de «l'agent» conçu comme porteur et reproducteur aveugle des exigences du système ou la formule de la liberté d'interprétation laissée à «l'acteur» dans un système de rôles. En sa forme pure, la première formule a toujours buté sur l'explication du changement, historiquement observable, des régimes de systématicité ou de l'éclatement des crises qui ne seraient pas inscrites dans la structure du système comme

7 Ibidem. 
l'est la contradiction motrice que doit supposer (chez Hegel ou Marx) la logique «dialectique» dans le fonctionnement de tout système; et la seconde formule qui fournit aujourd'hui en sociologie, en anthropologie et en histoire les concepts les plus utilisés pour rendre compte de l'innovation, dans les comportements ou les stratégies - s'est toujours heurtée à la difficulté d'expliquer la force des résistances au changement ou à l'innovation autrement que comme une hystérésis dépourvue de sens, de même que l'efficacité des normativités collectives autrement que comme une «contrainte » pesant de l'extérieur sur les choix du calculateur rationnel.

Mais la formule de l'actant, traité comme le régisseur des ressources d'action qui composent sa compétence, elle-même traitée comme un système, peut-elle être généralisée? Peut-on l'étendre à toute sociologie des formes et des forces symboliques dans la vie sociale voire à toute économie des transactions et des productions? Le problème méthodologique est celui que pose son extension au-delà des limites de la linguistique ou de la sémiologie. Son avantage à la fois théorique et programmatique est immédiatement visible, tant (I) sur la formule où l'acteur ne rencontre dans son environnement les systèmes institutionnels de règles ou de normes que comme des entraves à sa liberté d'improviser ou à son désir de maximiser son utilité, que (II) sur la formule où les systèmes semblent agir d'euxmêmes en tant qu'acteurs par le truchement d'agents-marionnettes.La formule d'un lien interne entre l'énonciation et les énoncés permet de décrire les «ressources » qu'un système sémiotique fournit à son «actant» lorsque celui-ci ne voit pas son action limitée de l'extérieur, c'est-à-dire lorsqu'il agit dans le cadre de sa liberté de discourir, disposant déjà des fins et des moyens de son discours par la maîtrise d'un système dont il est à la fois l'auteur et le chef d'orchestre, et jamais un simple exécutant. La formule est sans doute philosophiquement ambiguë, mais ce qui importe à la recherche c'est qu'elle est propice à une description des conduites qui ne réduit jamais leur dimension symbolique au vide ou à l'indétermination.

L'opéra, disait Toscanini, je crois, c'est est ce qui se passe quand un ténor et une soprano veulent coucher ensemble et qu'un baryton veut les en empêcher. D'une telle sémiotique d'un «genre» musical ou littéraire, on ne tire pas une sociologie de l'art susceptible de faire beaucoup de découvertes sur la sociologie ou l'histoire de l'opéra. C'est pourtant le programme de toute explication qui renferme son programme descriptif dans les bornes d'un système social, ne voulant connaître d'autre inventaire de ses rouages que celui qui figure dans le mode d'emploi de sa machinerie. Le programme qui décrit l'acteur par sa manière de jouer son rôle dans une intrigue mène-t-il la description plus loin? Cela n'est pas évident s'il dissuade de visiter les coulisses du théâtre ou s'il ne conduit, pas de scènes sociales en scènes sociales, à rencontrer des lieux d'interaction aménagés selon d'autres conventions ou d'autres interactions que celles qui s'opèrent sur une scène: on sait bien, depuis Clausewitz, qu'en tout affrontement l'exercice le plus physique de la violence est aussi un message adressé à l'adversaire; mais un message n'est jamais le tout des effets sociaux d'une violence qui n'est jamais purement symbolique. Le répertoire de l'opéra italien, le code des relations amoureuses dans une intrigue, et les règles de l'exécution vocale de la partition ne sont pas le tout de ce qui se joue entre les acteurs sur une scène d'opéra. Les exécutants y sont des acteurs par leur interprétation ou leur compétence, ils sont aussi des 
agents soumis, comme l'auteur, aux règles du système de l'opéra comme à celles qu'impose l'appartenance de ce système au système artistique et des fonctions de celui-ci dans la vie sociale et économique de l'époque; on n'en aurait jamais fini d'enquêter sur ce qu'ils font hors de l'opéra, sur leur passé scolaire ou familial par exemple, sur les rapports du public à la vie politique qui n'est jamais sans effet sur ses attentes par rapport au jeu théâtral, comme on le voit dans la réception des œuvres de Verdi. Le programme de l'actant propose au chercheur, par analogie avec l'étude des relations entre un «discours » et un «système linguistique », une ambition investigatrice d'une tout autre ampleur que celle qui découle de la description d'un jeu de rôles ou de la description des positions des agents dans un système. En somme, le programme de l'actant rend théoriquement pertinente la prospection des réalisations en nombre indéfini d'un acteur pour en extraire les opérations et les éléments en nombre fini qui peuvent en être reconstruites dans la syntaxe d'un ou plusieurs systèmes. Mais cette nouvelle analogie méthodologique, ici une métonymie, plutôt qu'une métaphore, dessine-t-elle un programme praticable par toutes les sciences sociales?

\section{Systèmes de signes et systèmes sociaux}

Il n'est pas étonnant que le système de la langue (ou plus généralement l'ensemble des systèmes de signes) se soit prêté à l'époque contemporaine à des renouvellements de la description et de l'interprétation scientifique qui ont pu aller dans la plupart des disciplines linguistiques jusqu'à la construction de «théories pures » ou de «modèles » formalisés. On a ainsi assisté à partir des années '50' sur la lancée des travaux des écoles formalistes et structuralistes de l'avant-guerre européenne et sur leur relance à partir des Etats-Unis à une diffusion rapide et longtemps enthousiaste dans toutes les sciences sociales des paradigmes issus d'une série de petites révolutions linguistiques en chaîne. Le "structuralisme», désignation élastique d'une théorie sans frontières stables, s'est diffusé vers l'anthropologie et la sociologie en y important les méthodes d'analyse structurale qui avaient si bien réussi dans les différents secteurs ou aux différents niveaux de l'analyse des langueset des discours: phonologie, morphologie, grammaire, sémantique, rhétorique, poétique etc. Mais le mouvement s'est ralenti puis arrêté à partir des années ' $80^{`}$ : mimétisme et décalques irréfléchis, a-t-on dit, exportation «sauvage» de méthodes et de concepts, simple mode, psittacisme même pour les abus conceptuels les plus criants dans ces plagiats sauvages. Que le langage d'une théorie puisse être galvaudé ne fait pas objection à sa valeur heuristique: il y a toujours eu dans les gazettes ou les salons tapage mondain et anodin, même autour des diffusions scientifiques les plus fécondes. Pourquoi alors, les sciences sociales n'ont-elle pas persisté bien longtemps dans leur effort pour devenir des sciences sémiotiques? N'y aurait-il pas des raisons intrinsèques à l'usure rapide du paradigme sémiotique ailleurs que sur ses terres de naissance? Et ces raisons ne tiendraient-elles pas à la texture même de ce que sont une langue naturelle ou un système de signes, cas trop privilégiés pour procurer une théorie adéquate du fonctionnement de tous les autres systèmes sociaux? Il faut convenir aujourd'hui que le schéma théorique de «l'actant symbolique», conçu comme partie intégrante du «système symbolique» qu'il fait fonctionner, ne fonctionne pas luimême, ou beaucoup plus approximativement, quand on l'applique à des systèmes 
sociaux où les interactions et les interdépendances entre éléments ne sont pas dotées de propriétés purement et exclusivement «symboliques».

La force descriptive et explicative du schème de l'actant s'affaiblit à mesure que l'on s'éloigne de la situation où les usagers d'un système de signes sont tous, également et indistinctement, les actants de sa mise en œuvre. Dans le cas de la pratique d'une langue naturelle, les actants sont agglomérés dans «la masse parlante », comme disait Saussure, qui se donnait ainsi un actant collectif, exempt de sociologie ou de psychologie, coextensif au système dès qu'il l'utilise. Bref le schème est inégalement réducteur des propriétés fonctionnelles et structurales des autres systèmes sociaux, puisque ceux-ci sont inégalement réductibles à de purs systèmes de communication. Le schème de la description linguistique, qui suppose toujours la possibilité d'une définition préalable des «unités sémantiques minimales » d'un système, fonctionne encore avec quelque profit descriptif dans l'analyse structurale des contes ou des mythes: chez Levi-Strauss, par exemple dans ses Mythologiques. Mais il y a déjà, dans les termes même de ce programme d'analyse, une certaine réduction du système culturel et social des sociétés dont l'auteur recense les structures mythiques pour n'en retenir que ce qui lui permet d'en isoler les «mythèmes » comme segments élémentaires pertinents pour la signification du récit. Le système décrit n'est plus alors que celui où, comme le dit l'auteur, «les mythes se pensent entre eux». Une telle sémiologie des structures contient-elle encore de l'explication sociologique ou anthropologique? On a, en tout cas, observé des autonomisations qui, emportées par la vague structuraliste, réduisaient bien plus abusivement les propriétés propres à des systèmes de comportements, ne les identifiant plus que par leur fonction sémantique d'expression ou d'échange de significations: après les «vestèmes» découpés par Barthes pour formuler sa recherche sur le système de la mode, on a eu les «gustèmes » dans la sociologie du goût, les «chorèmes »du géographe, etc. Il faut en convenir: dans le cas de systèmes sociaux qui sont à peine descriptibles comme des systèmes, tant ils sont intriqués à d'autres systèmes mêlant régularités institutionnelles et rencontres de séries causales indépendantes - c'est-à-dire dans la majorité des cas qui font l'objet du récit de l'historien, de la description du sociologue et de l'anthropologue, ou encore dans la comparaison historico-sociologique entre constellations singulières indécomposables, toutes démarches qui font la méthode commune des constructions typologiques de ces disciplines - le schéma de l'actant et de son système n'est plus guère applicable; en tout cas avec quelque utilité scientifique, autre que celle, bien sûr, de légitimer ses utilisateurs, dans la conjoncture, par leur ralliement à une avant-garde scientifique.

On pourrait vouloir dissocier ici le cas de l'économie de celui des autres sciences sociales parce qu'elle partage, à première vue, avec la linguistique la propriété de pouvoir isoler les éléments derniers des systèmes d'interdépendance qu'elle se donne pour objet (prix, quantités, relations d'équivalence dans l'échange), afin de raisonner ensuite sur eux en toute monosémie de définition et en toute rigueur de calcul. L'existence du «marché » comme lieu défini exclusivement par les transactions qui s'y déroulent fournit bien au calcul de l'économiste, comme le fait la «commutation» du sens des signifiants pour le linguiste, un moyen de procéder à la définition de ses «monèmes» par «description définie» de leurs traits pertinents. La phonologie moderne s'est fondée, avec l'Ecole de Prague, sur une décision de méthode qui rompait avec la forme classique de 
connaissance des langues - celle de la phonétique vouée à une description et à une mesure toujours plus fines de tous les traits observables dans les sons d'une «langue naturelle». La phonologie s'en tient volontairement à l'opération de «commutation» des signifiés pour construire, comme un système, le «tableau phonologique» des traits phonétiques pertinents - et de ceux-là seulement, soulignait Troubetzkoï ${ }^{8}$ - qui séparent les uns des autres les «phonèmes » dont la distinction par les locuteurs eux-mêmes permet l'intercompréhension du sens des mots dans l'utilisation quotidienne d'une langue naturelle. On voit immédiatement qu'aucun des systèmes sociaux qui font l'ordinaire des autres sciences sociales, dans leur travail de description empirique et de mise en ordre théorique d'un processus, ne dispose de la possibilité de faire «commuter» les propriétés de ses objets, puisque qu'elle ne dispose jamais du critère de «l'intercompréhension» du sens des messages, opération qui est de l'ordre d'une expérimentation véritable.

Dans les systèmes sociaux qui ne sont pas de purs systèmes d'échanges symboliques, l'isolation des éléments derniers est arbitraire, comme le sont toutes les constructions qui ont été tentées, à l'âge d'or du structuralisme sauvage, par les chercheurs pressés de «trouver» qui voulaient se donner un programme de description de systèmes ou de structures convenant à leur intuition intellectuelle, en toute indifférence aux protocoles de vérification empirique qu'appelait le langage de ce programme. On peut s'amuser à parler par métaphore d'une «grammaire générative » des attitudes ou des comportements, mais nul ne peut écrire les règles de cette grammaire comme on le fait de la grammaire d'un système de signes, sans parler de la description de sa sémantique. Science d'un système d'échanges qui n'est pas, de fait, un pur système d'inter-compréhension entre ses agents, l'économie est finalement plus proche en cela des autres sciences sociales que de la linguistique. Elle peut dialoguer avec une sociologie ou une histoire économiques, tandis que la linguistique ne peut dialoguer, sans déjuger sa théorie du langage, avec une sociologie du langage, tout au plus le peut-elle dans le cadre d'une sociolinguistique bâtie à cette fin sur un compromis laborieux.

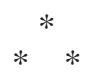

On retrouve finalement, comme toutes les fois où on examine le rapport épistémologique entre théories et protocoles empiriques dans l'histoire des sciences sociales, la forme particulière de scientificité qu'impose à la véridicité de leurs assertions la pluralité théorique de leurs langages de description, d'analyse et de preuve: c'est de cette pluralité théorique que découle, au cœur de chacune des sciences sociales, un pluralisme méthodologique qui explique aussi bien la fréquence de leurs hybridations transdisciplinaires que la virulence de leurs conflits interdisciplinaires. L'identité logique d'une science ne se reflète qu'éclatée ou déformée dans le miroir de son identité sociale. La description épistémologique et la description sociologique d'une science ne retrouvent pas la même science dans

8 N. S. Troubetzkoï, Principes de phonologie, (trad. J. Cantieneau), Paris, Klincksieck, 1949, p. 40. 
leurs creusets. C'est bien pourtant de la même science que l'on parle, qu'on la décrive comme un «système» de règles logiques ou comme une «intrigue » historique, reliant des acteurs au sein d'un réseau social de concurrence et d'échanges.

L'échec de l'utilitarisme à rendre compte des actions d'homo sociologicus aussi simplement que de celles d'homo æeconomicus ne dissipe finalement qu'une aspiration vaine, l'aspiration toujours déçue mais toujours vivace au cœur de chaque science sociale, à devenir une science aussi «dure» que les sciences exactes les plus «dures»: méthodologiquement unifiée, théorisée dans un «paradigme » unique, identiquement «protocolarisée » dans tous ses programmes d'observation, mathématisée en son langage et, pourquoi pas, formalisée de bout en bout. En économie, la domination durable, sous des formes à peine modifiée, d'une théorie comme celle de "l'équilibre général», associée à la persistance d'une épistémologie qui ne conçoit de théorie vraiment scientifique que la «théorie pure », semblent avoir confirmé les bénéfices de la révolution galiléenne qui a fondé la physique mathématique en ne retenant de l'observation du monde que les seules données susceptibles d'être transposées dans un calcul de «faits virtuels», en oubliant l'histoire et ses complexités singulières: ce qui n'a évidemment pas les mêmes conséquences pour expliquer la chute des corps ou la chute des empires, une valorisation boursière ou une floraison artistique. L'histoire et la sociologie, l'anthropologie ou l'archéologie ne se laissent pourtant pas oublier longtemps par les économistes qui prennent au sérieux la tâche explicative de leur discipline, surtout par les économistes les mieux avertis des pouvoirs et des limites du calcul utilitariste: Pareto aux origines du calcul à l'équilibre ou Arrow aujourd'hui'. La linguistique et la sémiologie, l'anthropologie et la démographie aussi, ont fait naître des espérances d'unification des langages théoriques ou de modélisation du devenir qui, comme les promesses du marxisme, du fonctionnalisme, du structuralisme ou de la grammaire générative, n'ont eu qu'un temps. L'histoire des sciences nous dissuade de confondre un main stream avec un paradigme de normalité scientifique, un moment de bonheur théorique avec une révolution scientifique au long cours.

\section{Ecole des Hautes Etudes}

en Sciences Sociales

Marseille

\footnotetext{
K.J. Arrow, « Economic history : A necessary though not sufficient condition for an économiste », The Theory of Economic Organization, 75 (2). Pareto a répété que le Traité de sociologie générale (1916), qui traite des conditions multiples et non-commensurables de «l'équilibre social »par une induction historique, englobait comme un cas plus simple le calcul des conditions de «l'équilibre économique» que le Cours d'économie politique ( 1896-1897) avait pu traiter par des équations mathématiques.
} 